\title{
Using Mobile Phones to Interact with Tabletop Computers
}

\author{
Christopher McAdam, Stephen Brewster \\ Glasgow Interactive Systems Group \\ School of Computing Science \\ University of Glasgow \\ Glasgow, G12 8QQ \\ www.dcs.gla.ac.uk/ chrism \\ \{christopher.mcadam, stephen.brewster\}@glasgow.ac.uk
}

\begin{abstract}
Tabletop computers can be used by several people at the same time, and many are likely to be carrying mobile phones. We examine different ways of performing interactions in this multi-device ecology. We conducted a study into the use of a phone as a controller for a dial manipulation task, comparing three different forms of interaction: direct touch, using the phone as a general-purpose tangible controller on a tabletop computer and manipulating the dial directly on the phone's screen. We also examined user performance for these interactions both with and without tactile feedback from the phone. We found interacting on the phone itself fastest overall, with tactile feedback improving performance. We also show a range of concerns that users have about using their phone as a controller. The results suggest that using a phone and table together can sometimes be better than using the table alone.
\end{abstract}

\section{Author Keywords}

Tabletop computer, mobile phone, tactile, Surface, dial

\section{ACM Classification Keywords}

H5.2 User Interfaces: Haptic I/O

\section{General Terms}

Human Factors

\section{INTRODUCTION}

Tabletop computers are increasingly used in public exhibits and displays. While they have yet to find their way into the home, public venues such as casinos, shops, hotels and museums are common locations to find tabletop computers. It is in spaces like these that the advantages of tabletops are easily seen, with their large size, 'walk-up-and-use' interfaces and multitouch input screens, allowing them to be used by several people at the same time.

It is very likely that each person at the tabletop will be carrying a mobile phone $[11,25]$. In this situation the phone can potentially be used as an additional way to interact with

Permission to make digital or hard copies of all or part of this work for personal or classroom use is granted without fee provided that copies are not made or distributed for profit or commercial advantage and that copies bear this notice and the full citation on the first page. To copy otherwise, or republish, to post on servers or to redistribute to lists, requires prior specific permission and/or a fee.

ITS 2011, November 13-16, Kobe, Japan.

Copyright 2011 ACM 978-1-4503-0871-7/11/11_..\$10.00. the tabletop $[14,18,21,24]$. When multiple devices are used, it is important to decide how these devices can be best used together. It could be assumed that interactions should occur on the tabletop, however, this may not be best; while the phone is smaller, its screen may be more precise than that of the tabletop. Depending on the context, the privacy offered by using the phone's screen instead of the public tabletop display may also be beneficial.

We can take advantage of the features of the phone for interaction. Mobile phones are capable of generating visual, audio and tactile feedback, and increasingly feature sensors such as accelerometers and magnetometers. Once paired with the tabletop, the phone can act as an 'active', generalpurpose tangible controller and the different capabilities of the phone can be leveraged to allow for a greater range of interactions to be performed. The phone can be used as a controller placed on the surface of the tabletop, but also in the space above the surface and away from the table. By providing users with additional feedback through the phone, it may be possible to improve performance in terms of the speed and accuracy of the interactions that they perform.

Our motivation is that users can use their phones as personal, general-purpose tangible controllers. As they will bring the phone with them to the tabletop and take it with them when they leave, this eliminates the need to prepare enough tangibles for each person and also the need to store the tangibles. As the phone has a range of inputs and outputs, it can be used to provide users with additional levels of feedback on interactions than they would get from a standard tangible controller. These inputs and outputs allow it to be used as a general-purpose tangible controller, instead of requiring several different tangibles that each performs only a single function.

There are many ways that phones can be used with tabletops. However, when there are multiple connected devices, it is not clear where the best place to perform the interaction would be. Should it always be performed on either the tabletop or the phone? Or is it dependent on the characteristics of the interaction it is? By exploring the different possibilities for performing interactions using a phone and a tabletop, we aim to determine if there are benefits to using both devices together, instead of only using the tabletop.

\section{BACKGROUND}


There are differences in user behaviour and performance when using tangibles verses direct touch. Terrenghi et al. [25] compared two versions of an interface on a tabletop: one that was entirely graphical and one that featured a tangible controller. They observed that, while user opinions on the different means of interaction were varied, the tangible controller was easier to use on average. Lucchi et al. [15] performed a comparison of the speed and error rates for touch and tangible interfaces. They note that the tangible interface was well suited for the spatial layout task used in their study and was much faster overall, but in certain conditions the touch interface could be superior (for example, using touch commands such as lasso selection to increase performance). The authors suggest that the tangibles performed better due to their graspable nature and users were better able to perform manipulation tasks because of this.

There is an increasing trend in the development of tangible controllers that are more than solid pieces of plastic. The haptic puck [17] provided interactive tactile feedback for tabletop computers. SLAP widgets [28] are a series of transparent tangible widgets for tabletop computers. They can be labelled dynamically using rear projection and several of the widgets provide a degree of tactile feedback, such as the sliders, buttons and keyboards. In studies of the knob control, Weiss et al. [28] found that their SLAP knob performed faster and with less overshoots of the target value compared to a virtual knob control, which they suggest was due to the SLAP version of the control requiring less visual attention to use. Patten et al. [20] describe a way of tracking objects on the tabletop that affords them a state that can be changed using physical dials attached to the object. This suggests it is useful to have dynamic, active tangible controllers providing richer feedback to the user.

While using a mobile phone to interact with a larger display is not very common, there have been some previous studies into this area. Shirazi et al. [24] produced a digital card game that included mobile phones and tabletop computers. Players were able to connect their phones to the tabletop via Bluetooth and use the phones as additional displays. They were also able to perform a series of gesture-based interactions with the phone to play the game. In their study they found that players preferred interacting using the phone and tabletop over using just the tabletop alone and that they found using the phone easier. Maunder et al. [18] describe a technique for allowing users to share media between their phones and public displays. Boring et al. [4] present Touch projector, a means for users to interact with remote screens using their mobile devices. They also provide techniques for improving user performance when interacting in this manner. Dachselt [6] describes a system for interactions between phones and large displays where users can navigate an interface on a large display by tilting the mobile phone. Content can also be transferred between the phone and display by performing 'throw' and 'fetch-back' gestures with the phone. Hardy et al. [8] describe a technique that allows a phone to act as a cursor controller for a public display and also for providing additional feedback to the user and extending the capabilities of the public display. They found that the combination of the devices, and the interplay between them, allowed for the exploration of 'richer' interaction techniques.

While most tangible controllers are designed for a specific task, other objects may be also suitable. Cheng et al. [5] describe using everyday objects, such as boxes, cylinders, sticks and CD cases, as tangible controllers, noting that these objects have been designed specifically for their own purposes and not for use as an input devices. As such, not every object may be suitable for use in this manner. They suggest that, while this may make an object inadequate for use as a major input device, the object's natural affordances could aid its use as an auxiliary controller. They found that using everyday objects in this manner was suitable in multitask scenarios and could be used to control background tasks such as music players. Everyday objects were found to be unsuitable for actions with unrecoverable results such as deletion. They were also not suitable for tasks that occurred with low frequency, but were suitable for use in situations where there was an intuitive mapping with physical affordances, such as rotating photos. This indicates that a mobile phone may be useful as a tangible controller but may not be suitable for every role, so it is important to determine where it would be most effective. Using phones as controllers on tabletops would offer up several benefits. Fiebrink et al. [7] noticed that participants treated the controller boxes used in their study as their own and were never used collectively or shared amongst users. Allowing users to use their phones as controllers would mean that their ownership is clearly established.

Mobile phones, and in particular smart phones, have been investigated for their potential as universal input devices [1] and using them as active tangible controllers on tabletop computers could allow for a wider range of interactions than would be found with standard tangible controllers. Handsense [29] is a way to determine which hand is holding a mobile device and how it is being held using capacitive sensing. This information could be used to increase the diversity of interactions that are possible when using a phone as a tangible controller. Having a phone paired to the tabletop could also free the interaction from the surface of the table itself. Hilliges et al. [9] present a method of allowing users to interact in the space above the surface of a tabletop computer, as well as directly on it. They demonstrate how this can allow for virtual objects to be 'picked up'. The proxemics [2] of the interaction between the phone and tabletop could be used to further refine how the phone can be used as a controller.

However, using phones in a nonstandard manner means that there are other issues that must be examined, such as security and privacy. Lucero et al. [16] note in their study shared collocated interactions with mobile phones that privacy is a concern. It is difficult to determine whether users 
would be happy to pass their own phone around a group from that study, as they did not use their own phones. Karlson et al. [13] examined the range of concerns that are exhibited when sharing mobile phones. This is important for us to consider, as we are suggesting the use of a mobile phone at a shared tabletop in a public setting, which increases the possibility that someone could pick up a phone that does not belong to him or her, either accidentally or on purpose. Phones typically offer an all-or-nothing approach to security, where the device is entirely locked and secure, or unlocked with everything accessible and, while users are happy to share their phones in certain circumstances, there is a wide diversity when it comes to the level of access that they are willing to let others have to the content on their phones.

\section{EXPERIMENT}

This paper presents an experiment investigating the use of mobile phones as general-purpose tangible controllers for simple interactions with tabletop computers. The goal is to investigate how to combine phones and tabletops, determining if it is feasible to use a mobile phone in this manner and to examine and contrast user performance using the phone with traditional direct-touch.

A Microsoft Surface (www.microsoft.com/surface) was used as the tabletop in this experiment. The mobile phone used was a Google Nexus One (www.google.com/phone /detail/nexus-one). Both of these devices are shown in Figure 1. These devices were connected to each other using Bluetooth. A byte tag was placed on the back of the phone, allowing it to be tracked when placed on the tabletop.
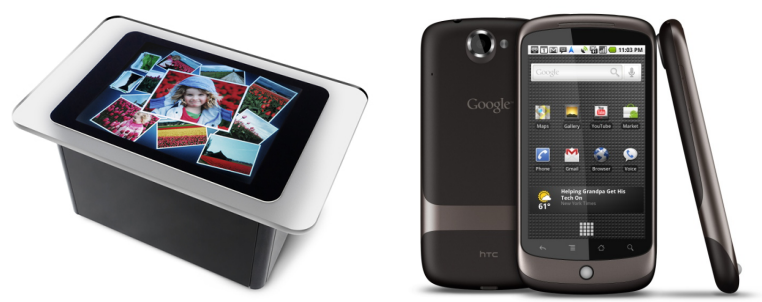

Figure 1: The equipment used in the experiment; Microsoft Surface with 30-inch display (left) and Google Nexus One with 3.7inch display (right).

In the experiment the participants performed a dial manipulation task. This task was chosen as dial control is a common use of tangible controllers on tabletops and rotation is one of the basic manipulations that tangibles afford $[3,15$, 24, 26-27]. The participants were presented with a dial and a target value. Their task was to set the dial to the specified value. Once the value was set, they would press the 'Next' button to move onto the next dial. They moved onto the next dial regardless of whether or not the current selection was correct. The experiment UI can be seen in Figure 2 and Figure 3.

\section{Methodology}

We compared user performance in the dial manipulation task using three different forms of interaction: direct touch, phone as a general-purpose tangible controller and interacting directly on the phone screen.

Direct touch: The dial is shown on the tabletop computer. The participant moves the dial by touching the black bar with a finger and dragging it to the desired value (see Figure 4).

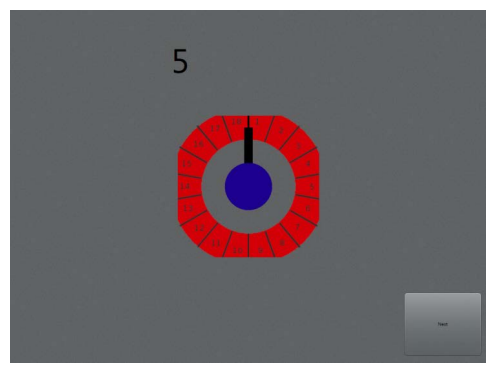

Figure 2: Screenshot of the experimental interface shown on the Microsoft Surface. The target value that the participant has to select is shown above the dial (which in this case has 18 segments) and the button to move onto the next dial is in the bottom-right corner.

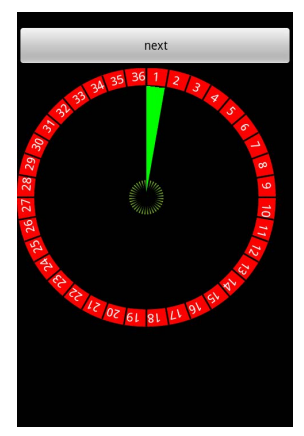

Figure 3: Screenshot of the experimental interface on the Nexus One for the Phone screen conditions. In these conditions, the selections made on the dial on the phone were also shown on the Surface.

General-Purpose Tangible controller: Here the dial on the Surface does not respond to finger touch control. Instead participants place the phone on the surface and rotate it to turn the dial (the phone placed in the centre of the dial on the table, oriented towards 0 , or 12:00 at the beginning of each trial; see Figure 5) Pointing direction is tracked via the byte tag on the back of the phone.

Phone screen: Here the dial on the table does not respond to touch or tangible control. A copy of the dial is shown on the phone's display. The participant sets the value of the dial by touching the position indicator on the phone's screen and dragging it to the desired position. The dial on the table changes value simultaneously with the dial on the phone (see Figure 6). There are no restrictions on how the participants can interact with the phone, for example they could interact using only one hand or with both hands. 
Tactile feedback has been shown to improve performance for interactions on touch-screen devices, such as for textentry $[10,19]$. Providing additional feedback using the vibration motor in the phone will augment the feedback present in the physical manipulations of the tangible controller.

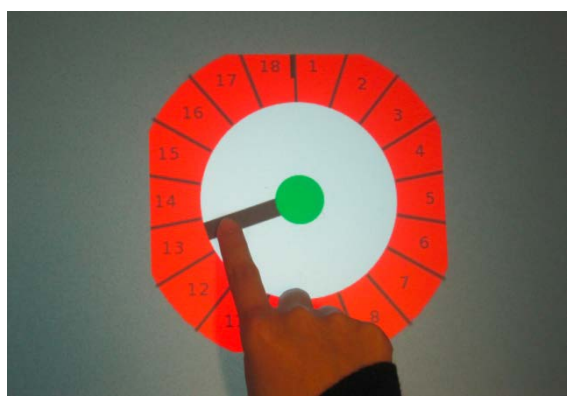

Figure 4: A participant makes a selection using Direct touch.
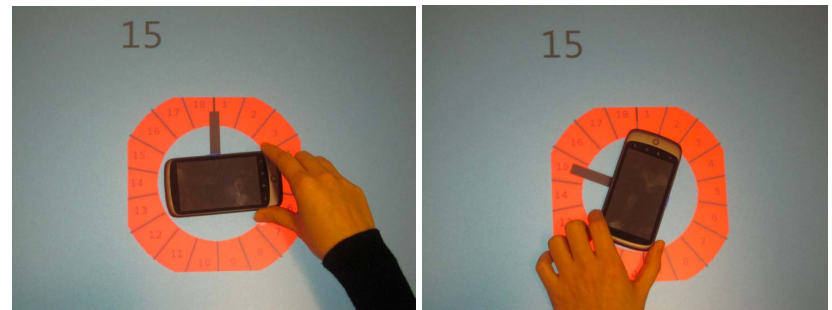

Figure 5: A participant makes a selection using the phone as a general-purpose tangible controller. The phone is placed on the tabletop oriented towards 12:00 at the beginning of each selection (left) and then the participant turns the phone to select the required value (right).

The experiment used a within-subjects design where the conditions (Table 1) were:

1. Interacting using Direct touch (DT);

2. Interacting using Direct touch with tactile feedback (DTF);

3. Interacting using the phone as a general-purpose Tangible controller (TC);

4. Interacting using the phone as a general-purpose Tangible controller with tactile feedback (TCF);

5. Interacting using the Phone screen (PT);

6. Interacting using the Phone screen with tactile feedback (PTF).

\begin{tabular}{cccc}
\hline ID & Feedback & Phone location & Dial Control \\
\hline DT & None & - & Direct touch \\
DTF & Tactile & Trouser pocket & Direct touch \\
TC & None & Tabletop & Phone tangible \\
TCF & Tactile & Tabletop & Phone Tangible \\
PT & None & In Hand & Phone screen \\
PTF & Tactile & In Hand & Phone Screen \\
\hline
\end{tabular}

Table 1: Summary of the features of the experimental conditions.

For the DTF condition, the tactile feedback was provided by the phone, which was placed in the participant's front trouser pocket (a common location for a phone to be kept). In this way they can receive the feedback from the tactile display - the phone - without having to hold it in their hands. For the other two tactile conditions (TCF and PTF), feedback was provided by the phone, which was held by the participant.

The dials were divided into varying numbers of segments (shown in Figure 7) to see if selection accuracy would be affected by target size. We used three different dials with 4 , 18 and 36 segments. The difference in target size $\left(90^{\circ}, 20^{\circ}\right.$ and $10^{\circ}$ respectively) allowed us to examine how accurate participants could be with the different interaction techniques and feedback, and how this would affect the time taken to complete the task. The dial always started at 0 and the marker could be moved both clockwise and counterclockwise, except at the two endpoints i.e. the dial will not turn from the lowest value directly to the maximum value, and vice versa. While the dials on the phone and table differed in diameter, they were the same in terms of segment placement, angular segment size and behaviour. The difference in diameter does not impact the selection of a target, as selection is an angular motion, and thus is consistent across the two devices. On both devices, the entirety of the marker bar is selectable, so the user is not required to move around the circumference of the dial to make a selection. The size of the dial is set depending on the characteristics of the method of interaction: On the phone the dial is bounded by the width of the screen, and on the tabletop it is set at a size that allows the phone to be placed in its centre without obscuring the segments or the dial marker.

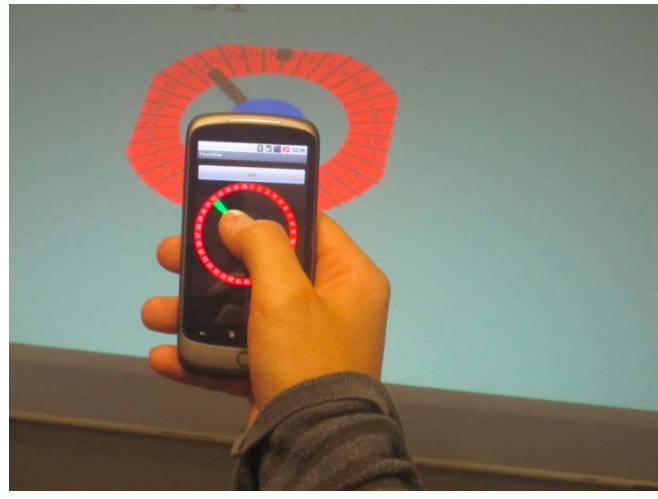

Figure 6: A participant makes a selection by interacting with the Phone screen. Here, a copy of the dial is shown on the phone screen and, as the participant changes the value of the dial on the phone, the changes are reflected on the dial on the tabletop. The target value is only displayed on the tabletop.

The experimental hypotheses were as follows:

1. Participants will achieve greater performance (time, error rate and subjective workload) with the general-purpose tangible controller than with direct touch (H1);

2. Participants will achieve greater performance (time, error rate and subjective workload) interact- 
ing via the phone screen than with the generalpurpose tangible controller (H2);

3. Participants will achieve greater performance (time, error rate and subjective workload) with the tactile feedback than without (H3).
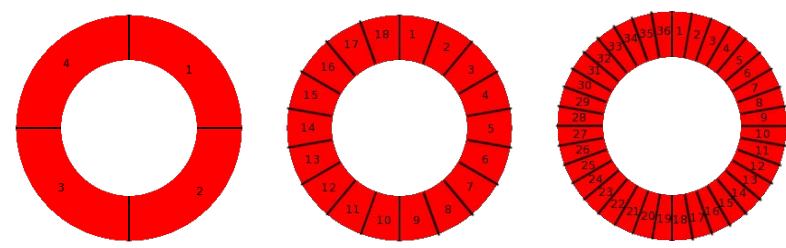

Figure 7: The three different dials used in the experiment (from left to right, 4 segments, 18 segments, 36 segments).

\section{Feedback Design}

In three of the six conditions tactile feedback was provided. This feedback was triggered when the user moved the position indicator from one segment of the dial to the next. For the direct touch and the general-purpose tangible controller, the feedback was a $250 \mathrm{~ms}$ vibration. For the phone screen condition, the feedback was a $50 \mathrm{~ms}$ vibration. The smaller duration of the feedback in this condition is due to the fact that the phone held in one hand while the other hand interacts directly with the screen, increasing the number of contact points with the phone and making it easier for the user to detect the feedback. In the other conditions, the phone will be placed in the users pocket or on the table, and we observed in our pilot studies that the longer feedback duration is necessary for clear detection, which we believe is due to the need to convey the feedback through layers of clothing, and the combination of fewer contacts on the phone and the friction of moving the phone against the surface of the tabletop. The feedback was generated using the vibration motor in the Nexus One.

The Bluetooth connection used between the Surface and the phone is a potential source of delay. We measured the time taken between sending a command from the surface to the point where the phone begins to vibrate. This was measured using Kaaresoja et al.'s Multimodal latency measurement tool [12]. We found that the time taken between command and onset of vibration was on average $145 \mathrm{~ms}$, and that the time taken between command and the phone reaching maximum vibration was $251 \mathrm{~ms}$.

\section{Experimental Procedure}

This experiment involved 13 participants ( 5 male, 8 female) aged between 21 and 30. All participants were university students and 6 had some previous experience of using a tabletop computer. All participants were seated during the experiment. There were no restrictions on how the participants could hold the phone and all participants were free to rest against the chair or the Surface if they wished.

The order of the conditions for each participant was randomised. For each condition, the participants were presented with a dial and a target value and were asked to set the dial to that value. Timing began when the participants first started manipulating the dial and stopped when they pressed the 'Next' button. They moved on to the next task regardless of whether or not the current selection was correct. There was no limit on how long they could take to make a selection. Participants made 60 selections in each condition, giving a total of $60 \times 6$, or 360 selections in total.

A training period was given before each condition to allow participants to familiarize themselves with the interaction and feedback provided. This training period consisted of the user making 10 selections on dials of varying segment size.

The dependent variables measured were: speed, accuracy and subjective workload (using the NASA TLX scales). At the end of the experiment participants were given a brief questionnaire to judge how acceptable they felt it was to perform these interactions in different hypothetical social situations. This is important as we are aware of how others perceive us in social situations and our willingness to perform certain actions is dependent on this [21]. An open interview followed this, which allowed participants to expand on why they felt this way and also to provide any other comments they wished about the interactions.

\section{RESULTS}

\section{Task Time}

The time taken for a participant to complete each selection was recorded. A Friedmans test showed there was a significant difference in the task time depending on which method of interaction was used $(\chi 2(2)=557.263, p<0.001$, see Figure 8). Post hoc analysis with Wilcoxon Signed-Rank Tests was conducted with a Bonferroni correction applied, resulting in a significance level of $\mathrm{p}<0.017$. There was a significant difference in the task time for direct touch vs. phone screen $(Z=4.139, \mathrm{p}<0.001)$, for tangible vs. phone screen $(Z=22.133, \mathrm{p}<0.001)$ and for direct touch vs. tangible $(Z=12.731, p<0.001)$. Selections were made fastest using the Phone Screen (mean $2072.62 \mathrm{~ms}$, SD 1273.72), followed by Direct Touch $(2535.71 \mathrm{~ms}$, SD 1259.21) and then Tangible (3174.31ms, SD 1605.38). This means Hypothesis 1 is rejected as the active tangible controller was slower than direct touch, but Hypothesis 2 is confirmed as the phone screen was the fastest interaction of the three.

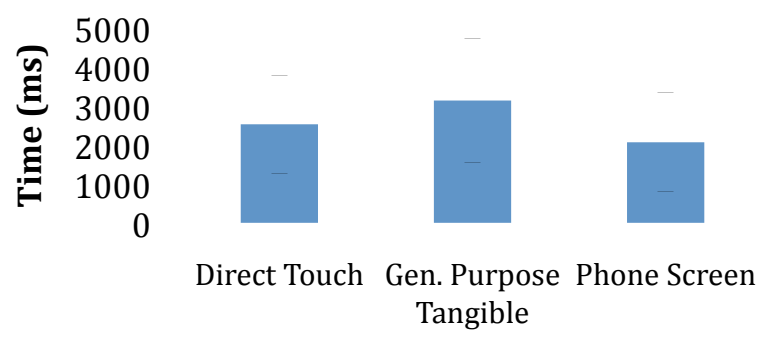

Figure 8: Average time taken for participants to complete a selection for each of the three forms of interaction, with standard deviations. Direct touch was sig. faster than general tangible, and phone screen was sig. faster than direct touch and general tangible. 
Analysis with Wilcoxon Signed-Rank Tests show that there was a statistically significant difference in the task time for feedback vs. no feedback $(Z=-3.849, \mathrm{p}<0.001)$ (Figure 9). Participants were faster on average when presented with tactile feedback $(2540.15 \mathrm{~ms}$, SD 1478.27) than they were without tactile feedback $(2630.28 \mathrm{~ms}$, SD 1433.24). This confirms Hypothesis 3.

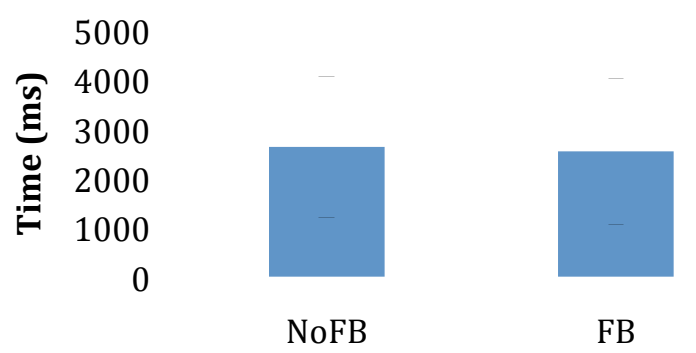

Figure 9: The average time taken for participants to complete a selection when presented with and without tactile feedback. Shown with standard deviations. Feedback was sig. faster then no feedback.

As expected, there was a statistically significant difference in the task time depending on how many segments were in the dial $\left(\chi_{2}(2)=1156.47, \mathrm{p}<0.001\right.$, see Figure 10). Post hoc analysis with Wilcoxon Signed-Rank Tests was conducted with a Bonferroni correction applied, resulting in a significance level set at $\mathrm{p}<0.017$. There was a statistically significant difference in the task time for dials with 4 segments vs. 18 segments $(Z=-20.947, p<0.001)$, dials with 4 segments vs. 36 segments $(Z=-29.273, p<0.001)$ and dials with 18 segments vs. 36 segments $(Z=-19.136, P<$ $0.001)$. Selections were made fastest on average on dials with 4 segments (1784.14ms, SD 976.87), followed by dials with 18 segments $(2575.41 \mathrm{~ms}$, SD 1368.98) and then dials with 36 segments $(3396.09 \mathrm{~ms}$, SD 1496.05). The smaller segments were harder to hit than the larger ones.

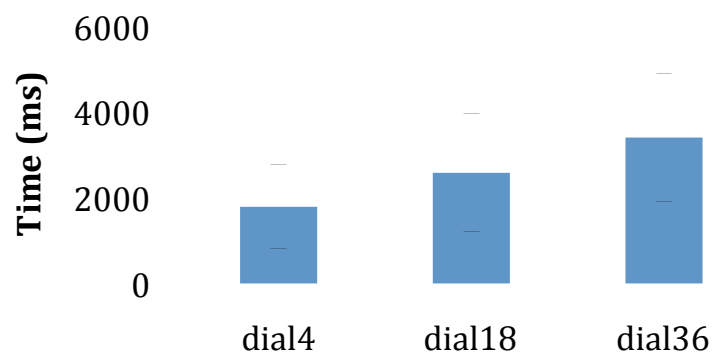

Figure 10: The average time taken for participants to complete a selection for each of the different dial sizes, shown with standard deviations. Dial4 was sig. faster than dial18 and dial 36, while dial18 was sig. faster than dial36.

These results partially confirm our hypotheses, with users performing faster using the phone than direct touch and the tangible controller, and were faster when presented with tactile feedback. However, users performed slower using the tangible controller than they did with direct touch.

\section{Selection Accuracy}

The accuracy of each selection was recorded. There was no statistically significant difference in the accuracy of selections across conditions: Phone Screen $(97.05 \%$ correct selections, SD 16.91), Direct Touch (96.34\%, SD 18.79), Tangible (95.76\%, SD 20.15).

When presented with tactile feedback, selections were made with an average accuracy of $96.31 \%$ (SD 18.85). When there was no tactile feedback, selections were $96.46 \%$ accurate (SD 18.49). Analysis with Wilcoxon Signed-Rank Tests show there was no statistically significant difference in the accuracy of selections for feedback vs. no feedback.

The accuracy of selection on dials with 36 segments was $97.05 \%$ (SD 16.91), on dials with 18 segments was $96.41 \%$ (SD 18.62) and on dials with 4 segments was $95.69 \%$ (SD 20.32). There was no statistically significant difference in the accuracy of selection depending on how many segments were in the dial.

The results for accuracy are less clear than for time. It may be that participants were trading accuracy against time and, in this case, kept their accuracy level high but slowing down with the different interaction techniques and feedback types.

\section{Subjective Workload}

The results of the NASA TLX questionnaire are shown in Figure 11. There was no significant effect for overall workload.

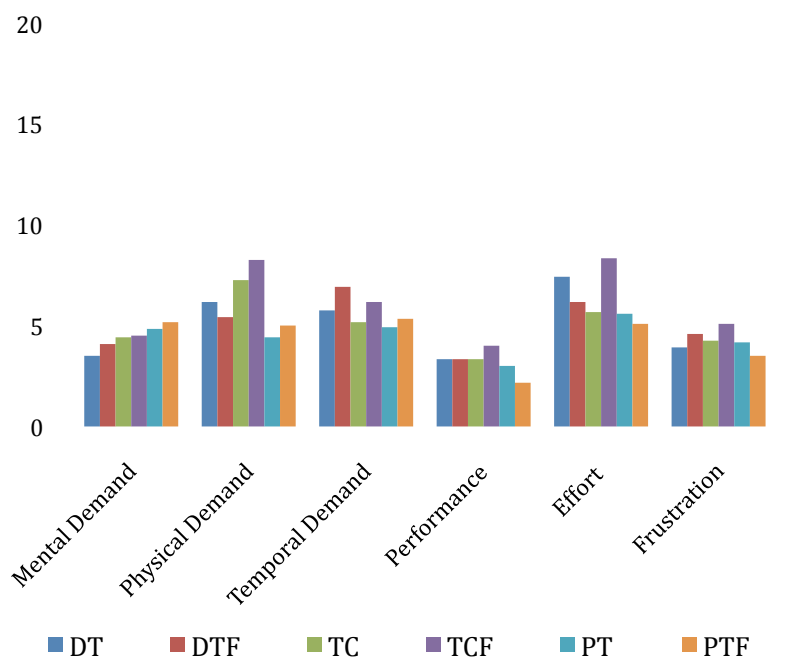

Figure 11: Average scores given by participants in the NASA TLX workload assessment. There was no significant difference for overall workload.

\section{Social Acceptability}

As this work proposes a non-standard use of mobile phones in public settings, it is important to not only measure the 
users' performance with these interactions, but also their opinions on whether or not they would be likely to use these interactions in the way that we propose. To that end, after completing the six conditions each participant was given a questionnaire to complete on the social acceptability of the interactions. Social acceptability concerns how acceptable people find it to perform particular actions or gestures in different contexts. They were asked to evaluate the acceptability in two different settings (home and public space) with three different social contexts for each (alone, with friends, with family, and alone, with friends, with strangers respectively). Rico [21] used this technique to evaluate gestural interactions in similar settings, as this questionnaire is based upon that work.

They were asked to score each interaction based, hypothetically, on how acceptable they felt it would be in that context. The values used in the scoring ranged from 0 (Totally unacceptable) to 5 (Perfectly acceptable).

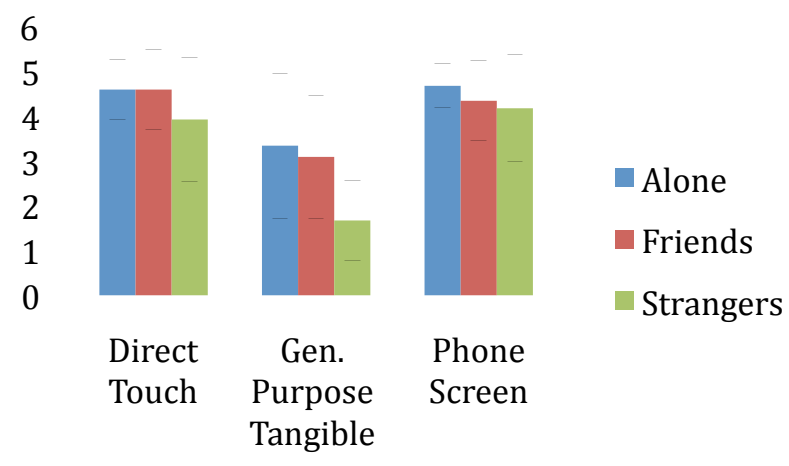

Figure 12: Average perceived acceptability of performing these interactions in a public space with three different social contexts (with strangers at the tabletop, with friends, and alone). Shown with standard deviations.

In a public setting (Figure 12), participants felt that using the phone as a tangible controller would be the least acceptable of the three forms of interactions. They also felt that each interaction would be less acceptable when there were strangers at the table.

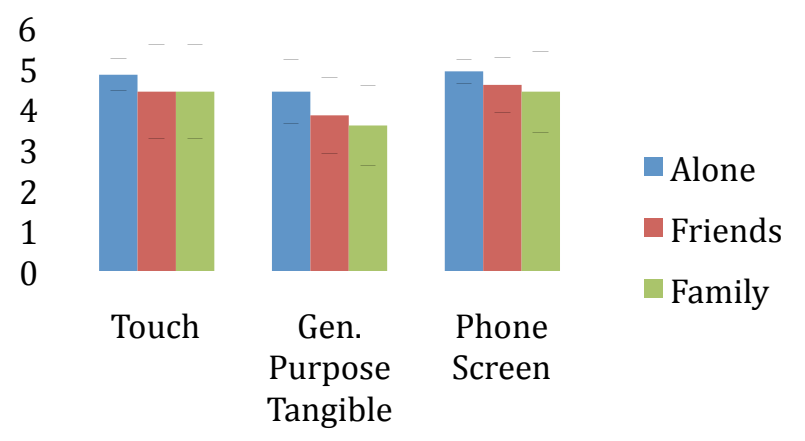

Figure 13: Average perceived acceptability of performing these interactions at home with three different social contexts (when alone, with friends, and with family), shown with standard deviations.

In a home setting (Figure 13) participants thought that using the phone as a tangible controller would be more acceptable than it in a public setting. The differences between the techniques in this setting were small. While they were happy to perform the interactions when they were on their own, they thought that it was slightly less acceptable to do so when they were with friends or family.

\section{Interview}

At the end of each session, each participant was interviewed to give them a chance to offer any thoughts they had about the different interactions and whether they would actually use them if they were available as features in a real situation. The interviewer took notes on the responses. The feedback received from these interviews was varied and showed a range of different opinions on the practicality of different aspects of the interactions. The opinions expressed on the different interactions are as follows:

\section{Direct touch}

Feedback: There were differing opinions on the feedback provided. While some participants felt the feedback was good and helped them to know if they were in the segment, others disliked it, feeling that there was too much of it and they were unsure of its usefulness. Others felt that the feedback was uncomfortable, with one participant describing it as ticklish. One participant felt that he might confuse the feedback from the tabletop with the vibrations the phone makes when it receives a text message.

Discretion: One thing that the participants particularly enjoyed was that receiving the tactile feedback through the phone was very discreet. Not needing to take your phone out of your pocket and being the only person who receives the feedback were felt to be benefits of this interaction.

Occlusion: Three participants felt that their hand was occluding the dial, making the selections difficult to perform at times.

\section{General-Purpose Tangible Controller}

Interaction: There were varied opinions on the usefulness of this interaction. While some felt that it was a slow means of control, that it was tiring and that clutching was a problem, others seemed to really enjoy it, saying that it was easier than direct touch and physically the easiest to perform overall. It was the preferred means of interaction for two participants.

Phone and tabletop together: Some participants felt that the phone and tabletop worked well as a unit in this way. But for others, the form factor of the phone made it more difficult, expressing that the phone felt heavy and that the size and bulk of the phone could make it an awkward controller, making the interaction more difficult than it should be.

Security: The security of this interaction was a major concern for participants. There were concerns raised over pri- 
vacy, as if you leave the phone on the tabletop then others could pick it up and see the contents. One participant noted it was an issue of trust, depending on who you were with. It was felt that it shows off your phone and could draw attention to pickpockets. Indeed, the chance that the phone could be stolen was the biggest security concern to the participants, but these concerns were often context dependent. Several participants stated that it would depend not only on what sort of venue you were in (a science museum versus a railway station, for example) but also on what country you were in. There was also a concern that they might forget the phone on the tabletop once they were finished.

Phone damage: Most participants highlighted concerns that one might damage the phone using it in this manner. In particular they were worried that the interactions could damage the phone case and lens cover, or that it may increase the chances of the phone being dropped.

\section{Phone Screen}

Ease of Use: In general participants found interacting directly on the phone's screen to be the easiest of the three techniques. It was described as feeling smoother than the other interactions by several participants. It was noted that you did not need to move much, and it was felt that you could go quite fast in making the selections. Participants felt that they were making smaller movements and less motion overall, and that it was very accurate. It was also noted as being easier to use the phone in this manner than as a tangible controller.

Social Use: It was noted that the etiquette of the interaction needs to be considered, as you are moving controls from a public shared space to a private one. Some liked the privacy that this would offer and others felt that this interaction would be the best to use if there was a large crowd at the tabletop. The benefits it offers for use in a public space may not be necessary in a home space and one participant felt it would be lazy to use the phone in this manner at home.

Familiarity with Phone: It was noted that the phone itself may override the interaction, for instance if it receives a call. There was also concern that the feedback provided would need to be clearly differentiated from those that the phone provides in its normal usage. It appears that the familiarity of using a mobile phone was one of the reasons why this interaction was so well liked, with one participant stating that, as she was more used to using her phone, it felt perfectly natural. However, this familiarity meant that one participant found the tactile feedback to be particularly distracting, as he is not used to his phone vibrating when he touches the screen. He suggested audio might provide better feedback for this reason. While touchscreen phones are very common, one participant felt that her finger was too big for the small screen on the phone.

Concentration: There were some differing opinions on how easy it was to concentrate when the interaction was split between the two devices. Having the device closer to you meant that some participants found it easier to concentrate, while others disliked having to split their focus between the two devices, observing that any distractions could cause errors.

Feedback: Several participants noted that they liked the tactile feedback that was provided, stating that it was helpful most of the time.

\section{General feedback}

There were some general thoughts on the feedback that applied to all three different interaction styles. Participants responded well to the feedback, finding it useful to know if they had moved into a new segment. The feedback also showed that the system was still responding. However, some participants did not find the feedback to be so useful. As they received a cue every time they moved into a new segment, they felt that they were receiving too much feedback, and as the experiment progressed they became used to it and 'stopped noticing it'.

Users are also very concerned about performing interactions that they feel could damage their mobile phone. Using the phone as a tangible controller on the tabletop in particular was highlighted as being potentially damaging to the phone. It was felt that moving the phone across the tabletop could damage the phone's case, and in particular the lens cover. It was also noted that if users are constantly placing their phone on the table and picking it up, then there is an increased chance that they may drop it. It is unlikely that users will perform any interactions that they feel could be damaging to their phone, so it is important to take this into consideration.

\section{DISCUSSION AND CONCLUSIONS}

The question of where to perform an interaction is an important one. When using a shared tabletop computer the display space of the tabletop is a valuable resource. Often people can be territorial about the space that they are using [23], with others often willing to wait their turn before using the tabletop, when there is no real need for them to do so. If it is possible to use phones to interact with tabletops, then this could make others more likely to participate at the shared tabletop. Our work shows that, for some interactions, using these devices together can be as good as or better than using the tabletop alone.

The experiment examined different forms of interaction for using dials on a tabletop and a mobile phone, looking to see where was the best location to perform the interaction. We found that using the phone as a general-purpose active tangible on the tabletop offered no improvements over direct touch (in fact, performed more slowly, contradicting one of our hypotheses) and that the best place to perform the interaction was directly on the phone screen, supporting one of our hypotheses. This suggests that in some cases it is best to offload tabletop controls to the phone and allow the interactions to be performed there. We only looked at one type of control, namely manipulating a dial. There are many differ- 
ent types of controls available and further studies will show what the best way to combine phone and tabletop interactions would be in these cases. It is also necessary to examine ways of switching between two or more different controls, to see if the phone can act as a single general-purpose controller.

There are also many different ways of using a mobile phone and a tabletop together. For example, when using the phone as a general-purpose tangible controller, the phone is not limited to interaction on the table's surface but can be used in the space above and around the tabletop. The different sensors of the mobile phone could be leveraged to provide more information about the orientation of the controller, and possibly how it is being held, allowing more degrees of interaction between the phone and the tabletop.

It is important for designers of tabletop systems to consider the use of additional devices in the design of their systems. Using these devices together could offer improved performance, or a better overall experience, than using the tabletop alone, opening up new avenues of interaction. For tabletops in public settings, this would mean that users of the table would be able to use their phone as a private interaction space and the tabletop as a shared, public space. They could then input passwords or look up private data such as emails, without the others at the tabletop being able to see. When looking at these multi-device ecologies, it is necessary to take the capabilities of each device into account in order to use them together in the best way. Our study shows that while using the phone as a tangible controller did not improve performance, interacting via the phones screen did. There are likely other ways that the phone can be used in conjunction with the tabletop to improve the experience for the user, and more work will be carried out to explore this further.

Users had several concerns about using their phones on tabletops. Security is an important issue; users were worried that using the phone in this manner could draw attention to pickpockets and thieves. However, the fear of this was very context dependent. Several participants stated that this would depend on the type of place that they were in. For example, in a university library you will typically find a subset of the population, and they will most likely be there for a specific reason. It may also feel more secure if security guards are present. However, in places such as railway stations and shopping centres, which are open to everyone, there was more concern that the phone may be stolen. The people with you at the tabletop also play a factor in these concerns, with the participants feeling it was less acceptable to perform these interactions when there were people they did not know using the tabletop. This context was not limited to the type of venue, but also to the country that you were in. Some participants said that while they were worried about their phone being stolen from the tabletop if they were in the United Kingdom, they would not be concerned about it in Singapore. They stated that this was because in the public food courts in Singapore, it is common to leave your bags/phone/jackets at one of the tables to reserve it as yours, before going to get your food. Clearly the social contexts in which the interactions are important for determining what is acceptable, and this must be considered very carefully when designing interactions.

Providing the users with tactile feedback improved their performance in the tasks. While one of the benefits of tangible controllers is that they offer inherent tactile feedback, this shows that this feedback can be enhanced even further, and that providing as much feedback to the user as possible is beneficial to them.

In conclusion, we examined different ways in which a mobile phone can be used in conjunction with a tabletop computer and found that users performed best when using the phone directly to control the tabletop, able to make selections in approx $66 \%$ of the time it took with the phone as a general-purpose tangible controller, and in approx. $80 \%$ of the time it took using direct touch, while maintaining the same level of accuracy. Further we found that users performed faster when presented with tactile feedback than they did without. We also examined the acceptability of these interactions in different social contexts. When dealing with tabletops in public spaces, with multiple users, this means that we need to look beyond the tabletop to all the different devices in that environment, from mobile phones to tablets, and examine how these devices can be used in conjunction with each other, utilizing the abilities of each device, to provide the best experience for the user.

\section{ACKNOWLEDGMENTS}

This research was funded by an EPSRC DTA. The Microsoft Surface used in this experiment was provided by the SFC MATCH project.

\section{REFERENCES}

1. Ballagas, R., Borchers, J., Rohs, M. and Sheridan, J. G. The Smart Phone: A Ubiquitous Input Device. IEEE Pervasive Computing, 5, 1 2006), 70.

2. Ballendat, T., Marquardt, N. and Greenberg, S. Proxemic interaction: designing for a proximity and orientation-aware environment. Proceedings ITS2010. ACM Press, p 121-130.

3. Baudisch, P., Becker, T. and Rudeck, F. Lumino: tangible blocks for tabletop computers based on glass fiber bundles. Proceedings of ACM CHI 2010. ACM Press, pp1165-1174

4. Boring, S., Baur, D., Butz, A., Gustafson, S. and Baudisch, P. Touch projector: mobile interaction through video. Proceedings of ACM CHI 2010. ACM Press, pp2287-2296.

5. Cheng, K.-Y., Liang, R.-H., Chen, B.-Y., Laing, R.-H. and Kuo, S.-Y. iCon: utilizing everyday objects as ad- 
ditional, auxiliary and instant tabletop controllers. Proceedings of ACM CHI 2010. ACM Press, pp1155-1164.

6. Dachselt, R. and Buchholz, R. Natural throw and tilt interaction between mobile phones and distant dis-plays. Proceedings of ACM CHI 2009. ACM press, pp32533258

7. Fiebrink, R., Morris, D. and Morris, M. R. Dynamic mapping of physical controls for tabletop groupware. Proceedings of ACM CHI 2009. ACM Press, pp471-480

8. Hardy, R. and Rukzio, E. Touch \& Interact: Touchbased Interaction with a Tourist Application. Proceedings of MobileHCI '08. ACM Press, pp 531-534.

9. Hilliges, O., Izadi, S., Wilson, A. D., Hodges, S., Garcia-Mendoza, A. and Butz, A. Interactions in the air: adding further depth to interactive tabletops. Proceedings of UIST 2009. ACM Press, pp139-148.

10. Hoggan, E., Brewster, S. A. and Johnston, J. Investigating the effectiveness of tactile feedback for mobile touchscreens. Proceedings of ACM CHI 2008. ACM Press, pp1573-1582

11. Ichikawa, F., Chipchase, J. and Grignani, R. Where's The Phone? A Study of Mobile Phone Location in Public Spaces. City, 2005.

12. Kaaresoja, T. and Brewster, S. Feedback is... late: measuring multimodal delays in mobile device touchscreen interaction. Proceedings of ICMI-MLMI 2010. ACM Press.

13. Karlson, A. K., Brush, A. J. B. and Schechter, S. Can i borrow your phone?: understanding concerns when sharing mobile phones. Proceedings of ACM CHI 2009. ACM Press, pp1647-1650.

14. Kray, C., Nesbitt, D., Dawson, J. and Rohs, M. Userdefined gestures for connecting mobile phones, public displays, and tabletops. Proceedings of MObileHCI 2010. ACM Press, pp239-248.

15.Lucchi, A., Jermann, P., Zufferey, G. and Dillenbourg, P. An empirical evaluation of touch and tangible interfaces for tabletop displays. Proceedings of TEI 2010. ACM Press, pp177-184.

16. Lucero, A., Holopainen, J. and Jokela, T. Pass-themaround: collaborative use of mobile phones for photo sharing. Proceedings of CHI2011. ACM Press, p17871796.

17. Marquardt, N., Nacenta, M. A., Young, J. E., Carpendale, S., Greenberg, S. and Sharlin, E. The Haptic Tabletop Puck: tactile feedback for interactive tabletops. Proceedings of ITS 2009. ACM Press, p85-92.
18. Maunder, A., Marsden, G. and Harper, R. Creating and sharing multi-media packages using large situated public displays and mobile phones. Proceedings of MobileHCI2007. ACM Press, p222-225.

19. McAdam, C. and Brewster, S. Distal tactile feedback for text entry on tabletop computers. Proceedings of BCS HCI 2009. British computer Society, p504-511.

20.Patten, J., Ishii, H.,Hines, J. and Pangaro, G.. Sensetable: a wireless object tracking platform for tangible user interfaces. Proceedings of CHI2001. ACM Press, p253-260.

21. Rico, J. and Brewster, S. Usable gestures for mobile interfaces: evaluating social acceptability. Proceedings of ACMCHI 2011. ACM Press, p887-896.

22. Schmidt, D., Chehimi, F., Rukzio, E. and Gellersen, H. PhoneTouch: a technique for direct phone interaction on surfaces. Proceedings of UIST 2010. ACM Press, p1316.

23. Scott, S. D., Sheelagh, M., Carpendale, T. and Inkpen, K. M. Territoriality in collaborative tabletop workspaces. Proceedings of CSCW2004. ACM Press, p294-303.

24. Shirazi, A. S., Parvahan, P., Ahrens, B. and Schmidt, A. Poker Surface: Combining a Multi-Touch Table and Mobile Phones in Interactive Card Games. Proceedings of MobileHCI 2009. ACM Press, p1-2.

25. Terrenghi, L., Kirk, D., Richter, H., Kr, S., Hilliges, O. and Butz, A. Physical Handles at the Interactive Surface: Exploring Tangibility and its Benefits. Proceedings of AVI 2008. ACM Press, p138-145.

26. Tryhorn, C. Developing countries drive explosion in global mobile phone use, http:/www.guardian.co.uk/business/2009/mar/02/mobil e-phone-internet-developing-world, 2009, 03/03/2009 2009).

27. Tuddenham, P., Kirk, D. and Izadi, S. Graspables revisited: multi-touch vs. tangible input for tabletop displays in acquisition and manipulation tasks. Proceedings of ACM CHI 2010. ACM Press, p2223-2232.

28. Weiss, M., Wagner, J., Jansen, Y., Jennings, R., Khoshabeh, R., Hollan, J. D. and Borchers, J. SLAP widgets: bridging the gap between virtual and physical controls on tabletops. Proceedings of ACM CHI 2009. ACM Press, pp3229-3234.

29. Wimmer, R. and Boring, S. HandSense: discriminating different ways of grasping and holding a tangible user interface. Proceedings of TEI 2009. ACM Press, p359362. 